\title{
HOW TO DISTINGUISH BETWEEN CUBIC AND ISOTROPIC CRITICAL BEHAVIOURS
}

\author{
D. V. Pakhnin, A. I. Sokolov \\ Saint Petersburg Electrotechnical University \\ 5 Professor Popov Str., Saint Petersburg 197376, Russia
}

(Received September 21, 2001)

\begin{abstract}
For the three-dimensional cubic model, the nonlinear susceptibilities of the fourth, sixth, and eighth orders are analyzed and the parameters $\delta^{(i)}$ characterizing their reduced anisotropy are evaluated at the cubic fixed point. The anisotropy parameters are found to be: $\delta^{(4)}=0.054 \pm 0.012$, $\delta^{(6)}=0.102 \pm 0.02$, and $\delta^{(8)}=0.144 \pm 0.04$, indicating that the anisotropic (cubic) critical behaviour predicted by the advanced higher-order renormalization-group analysis should be, in principle, visible in physical and computer experiments.

Key words: renormalization group, cubic model, nonlinear susceptibilities, universal coupling constants, four-loop expansions.
\end{abstract}

PACS number(s): 64.60.Ak, 11.10.Lm, 64.60.Fr, 75.40.Cx

Thirty years ago Wilson and Fisher discovered that in the vicinity of the critical point the order parameter fluctuations may change the effective anisotropy of the system drastically [1]. Having studied the anisotropic $X Y$ model within the $\epsilon$ expansion they found that approaching $T_{c}$ this model either becomes effectively isotropic or developes its anisotropy further [1] until the fluctuationinduced first-order phase transition takes place $[2,3]$. What scenario the system chooses depends on the value and sign of the anisotropic coupling constant in the initial Landau-Wilson Hamiltonian. In the course of studying the generalized cubic model, the crucial role of the order parameter dimensionality $n$ was revealed: for $n<n_{c}$ the model undergoing the continuous phase transition demonstrates isotropic critical behaviour while for $n>n_{c}$ it remains anisotropic at criticality [4]. The numerical value of the marginal spin dimensionality $n_{c}$ separating these two regimes is of prime physical importance since it fixes the true mode of the critical behaviour of real cubic ferromagnets and of some other systems of interest.

First estimates of $n_{c}$ for the cubic model obtained from the lower-order renormalization-group (RG) expansions $[3,5,6]$ turned out to be in favour of the inequality $n_{c}>3$ indicating that cubic ferromagnets should belong to the class of universality of the three-dimensional (3D) Heisenberg model. However, the resummation of the three-loop RG series [7,8] and subsequent higherorder RG calculations performed both in three [9-11] and $(4-\epsilon)$ [12] dimensions shifted the numerical estimate for $n_{c}$ downwards fixing it below $3[7-18]$. Remarkable consensus between different field-theoretical approaches and resummation techniques was achieved in the course of this study: 3D RG calculations [11], resummed $\epsilon$ expansion [15], biased $\epsilon$ expansion technique [11], and pseudo$\epsilon$ expansion analysis [17] lead to $n_{c}=2.89, n_{c}=2.855$, $n_{c}=2.87$, and $n_{c}=2.862$, respectively. Hence, according to the update, most accurate theoretical data, cubic ferromagnets should demonstrate the specific-cubic-critical behaviour with special set of critical exponents.

Since $n_{c}$ is very close to the physical value $n=3$, the cubic fixed point lies very near the Heisenberg one at the RG flow diagram and the critical exponents for both fixed points almost coincide. For instance, the susceptibility exponent $\gamma$ is equal to $1.3895(50)$ for the $3 \mathrm{D}$ Heisenberg model [19] while at the cubic fixed point $\gamma=1.390(6)[11]$. It is clear that measuring the critical exponents one can not distinguish between the cubic and Heisenberg critical behaviours.

In such a situation, some alternative physical quantities should be addressed to clear up how the systems with the cubic symmetry behave approaching $T_{c}$. In this paper, the nonlinear susceptibilities of the $3 \mathrm{D} \mathrm{cu}^{-}$ bic model are studied in the framework of the fieldtheoretical RG approach, with a particular attention paid to their anisotropy at criticality. It will be shown that the anisotropy of nonlinear susceptibilities is sensitive to the type of critical asymptotics and its measurement can be used, in principle, for detection of the cubic (non-Heisenberg) critical behaviour, provided the anisotropy is as strong as the higher-order RG calculations predict.

In the critical region, the expansion of the free energy of the cubic model in powers of the magnetization components $M_{\alpha}$ may be written in the form:

$$
\begin{aligned}
& F\left(M_{\alpha}, m\right)=F(0, m)+\frac{1}{2} m^{2-\eta} M_{\alpha}^{2} \\
& +m^{1-2 \eta}\left(u_{4}+v_{4} \delta_{\alpha \beta}\right) M_{\alpha}^{2} M_{\beta}^{2} \\
& +m^{-3 \eta}\left(u_{6}+q_{6} \delta_{\alpha \beta}+v_{6} \delta_{\alpha \beta} \delta_{\alpha \gamma}\right) M_{\alpha}^{2} M_{\beta}^{2} M_{\gamma}^{2}+\ldots
\end{aligned}
$$

where $\eta$ is a Fisher exponent, $m$ being an inverse correlation length, and $u_{4}, v_{4}, u_{6}, q_{6}, v_{6}$ are dimensionless effective coupling constants acquiring, under $T \rightarrow T_{c}$, certain universal values. These coupling constants are related to the nonlinear susceptibilities defined in a conventional 
way:

$$
\begin{aligned}
& \chi_{\alpha \beta \gamma \delta}^{(4)}=\left.\frac{\partial^{3} M_{\alpha}}{\partial H_{\beta} \partial H_{\gamma} \partial H_{\delta}}\right|_{H=0}, \\
& \chi_{\alpha \beta \gamma \delta \mu \nu}^{(6)}=\left.\frac{\partial^{5} M_{\alpha}}{\partial H_{\beta} \partial H_{\gamma} \partial H_{\delta} \partial H_{\mu} \partial H_{\nu}}\right|_{H=0} .
\end{aligned}
$$

Of prime importance are the nonlinear susceptibilities in two particular cases, when (i) an external magnetic field is parallel to a cubic axis $\left(\chi_{c}^{(i)}\right)$ and (ii) it is oriented along a cell space diagonal $\left(\chi_{d}^{(i)}\right)$. For these two highly symmetric directions the differences between corresponding values of nonlinear susceptibilities are known to be maximal, i. e., the anisotropy is most pronounced. It is easy to show that

$$
\begin{gathered}
\chi_{c}^{(4)}=-24 \frac{\chi^{2}}{m^{3}}\left(u_{4}+v_{4}\right) \\
\chi_{d}^{(4)}=-24 \frac{\chi^{2}}{m^{3}}\left(u_{4}+\frac{v_{4}}{3}\right) . \\
\chi_{c}^{(6)}=720 \frac{\chi^{3}}{m^{6}}\left[8\left(u_{4}+v_{4}\right)^{2}-u_{6}-q_{6}-v_{6}\right], \\
\chi_{d}^{(6)}=720 \frac{\chi^{3}}{m^{6}}\left[8\left(u_{4}+\frac{v_{4}}{3}\right)^{2}-u_{6}-\frac{q_{6}}{3}-\frac{v_{6}}{9}\right] .
\end{gathered}
$$

where $\chi$ is a linear susceptibility. To characterize the anisotropy strength, we define the reduced parameters

$$
\delta^{(4)}=\frac{\left|\chi_{c}^{(4)}-\chi_{d}^{(4)}\right|}{\chi_{c}^{(4)}}, \quad \delta^{(6)}=\frac{\left|\chi_{c}^{(6)}-\chi_{d}^{(6)}\right|}{\chi_{c}^{(6)}}
$$

Below, they will be estimated at criticality.

Let us start from the lower-order nonlinear susceptibility $\chi^{(4)}$. The coordinates of the cubic fixed point $u_{4}^{*}$ and $v_{4}^{*}$ in three dimensions are known from the higherorder RG calculations, with resummed four-, five-, and six-loop RG expansions yielding very close numerical results $[9,11]$. Considering recent six-loop RG estimates as the most reliable ones, we accept $u_{4}^{*}=0.755 \pm 0.010$, $v_{4}^{*}=0.067 \pm 0.014$ [11]. The substitution of these num- bers into Eqs. (3), (5) gives

$$
\delta^{(4)}=0.054 \pm 0.012
$$

To determine $\chi_{c}^{(6)}, \chi_{d}^{(6)}$, and $\delta^{(6)}$, we have to calculate the effective coupling constants $u_{6}, q_{6}$, and $v_{6}$. They will be found perturbatively, using the field-theoretical RG approach in three dimensions. Our analysis is based on the well-known Landau-Wilson Hamiltonian of the 3D $n$-vector cubic model:

$$
H=\frac{1}{2} \int d^{3} x\left[m_{0}^{2} \varphi_{\alpha}^{2}+\left(\nabla \varphi_{\alpha}\right)^{2}+\frac{u_{0}}{12} \varphi_{\alpha}^{2} \varphi_{\beta}^{2}+\frac{v_{0}}{12} \varphi_{\alpha}^{4}\right],
$$

All the RG calculations are carried out within a massive theory under zero-momenta normalizing conditions for the renormalized Green function $G_{R}(p, m)$ and the four-point vertices $U_{R}\left(p_{i}, m, u, v,\right), V_{R}\left(p_{i}, m, u, v,\right)$ :

$$
\begin{aligned}
& G_{R}^{-1}(0, m)=m^{2},\left.\quad \frac{\partial G_{R}^{-1}(p, m)}{\partial p^{2}}\right|_{p^{2}=0}=1, \\
& U_{R}(0, m, u, v)=m u, \quad V_{R}(0, m, u, v)=m v .
\end{aligned}
$$

Here, the value of the one-loop vertex graph including the factor $(n+8)$ is absorbed in $u$ and $v$. Quartic effective couplings $u$ and $v$ thus defined are related to $u_{4}$ and $v_{4}$ entering Eqs. (1), (3), (4) in an obvious way:

$$
u=\frac{n+8}{2 \pi} u_{4}, \quad v=\frac{n+8}{2 \pi} v_{4}
$$

We limit ourselves by the four-loop RG approximation that proved to lead to quite a good numerical estimate for the universal value of the sextic effective coupling for the 3D $O(n)$-symmetric model $[20,21]$. Since the symmetry factors and integrals for all the relevant Feynman diagrams have been found earlier [21], what we have to do is the calculation of the tensor (field) factors for 6point vertex graphs and mass insertions generated by the $O(n)$-symmetric and cubic interactions. Performing this calculation and then renormalizing the "bare" perturbative expansions for the 6-point vertices precisely in the same way as it has been done for the isotropic $n$-vector model [20,21], we arrive at following four-loop RG expansions:

$$
\begin{aligned}
u_{6}= & \frac{8 \pi^{2}}{3} \frac{u^{2}}{(n+8)^{3}}\left[(n+26) u+9 v-\frac{1}{(n+8)}\left(\frac{34 n+452}{3} u^{2}+124 u v+18 v^{2}\right)\right. \\
& +\frac{1}{(n+8)^{2}}\left[\left(1.065025 n^{2}+157.42454 n+1323.0960\right) u^{3}+(19.382741 n\right.
\end{aligned}
$$




$$
\begin{aligned}
& \left.+1873.9825) u^{2} v+(2.3101121 n+679.33934) u v^{2}+100.90652 v^{3}\right] \\
& -\frac{1}{(n+8)^{3}}\left[\left(-0.0638 n^{3}+52.451 n^{2}+2314.99 n+14387.6\right) u^{4}\right. \\
& +\left(-2.3434 n^{2}+1006.56 n+29601.0\right) u^{3} v+(100.156 n+18985.4) u^{2} v^{2} \\
& \left.\left.+(11.286 n+5872.61) u v^{3}+782.865 v^{4}\right]\right] \text {. } \\
& q_{6}=\frac{8 \pi^{2}}{3} \frac{u v}{(n+8)^{3}}\left[72 u+27 v-\frac{1}{(n+8)}\left((4 n+520) u^{2}+468 u v+108 v^{2}\right)\right. \\
& +\frac{1}{(n+8)^{2}}\left[\left(-1.1357879 n^{2}+149.54004 n+5366.1581\right) u^{3}\right. \\
& \left.+(-32.454138 n+8267.9231) u^{2} v+4107.2194 u v^{2}+775.79040 v^{3}\right] \\
& -\frac{1}{(n+8)^{3}}\left[\left(0.3986 n^{3}-19.2521 n^{2}+3351.86 n+66591.7\right) u^{4}\right. \\
& +\left(11.6195 n^{2}+115.223 n+148119\right) u^{3} v+(-419.561 n+119322) u^{2} v^{2} \\
& \left.\left.+46560.3 u v^{3}+7520.89 v^{4}\right]\right] \text {. } \\
& v_{6}=\frac{8 \pi^{2}}{3} \frac{v^{2}}{(n+8)^{3}}\left[54 u+27 v-\frac{1}{(n+8)}\left[(-6 n+492) u^{2}+540 u v+162 v^{2}\right]\right. \\
& +\frac{1}{(n+8)^{2}}\left[\left(1.3828190 n^{2}-50.171743 n+5947.5257\right) u^{3}\right. \\
& \left.+(-88.612482 n+10696.342) u^{2} v+6632.1372 u v^{2}+1481.5855 v^{3}\right] \\
& -\frac{1}{(n+8)^{3}}\left[\left(-0.4873 n^{3}+6.2132 n^{2}+114.760 n+83872.7\right) u^{4}\right. \\
& +\left(25.5088 n^{2}-2440.17 n+212729\right) u^{3} v+(-1281.62 n+205263) u^{2} v^{2} \\
& \left.\left.+93009.1 u v^{3}+16755.0 v^{4}\right]\right] \text {. }
\end{aligned}
$$

These RG expansions should obey some exact relations appropriate to the systems with cubic anisotropy. Indeed, for $n=2$ model ( 7 ) is known to possess the specific symmetry property. If the field $\varphi_{\alpha}$ undergoes the transformation

$$
\begin{aligned}
& \varphi_{1} \rightarrow\left(\varphi_{1}+\varphi_{2}\right) / \sqrt{2}, \\
& \varphi_{2} \rightarrow\left(\varphi_{1}-\varphi_{2}\right) / \sqrt{2},
\end{aligned}
$$


quartic coupling constants are also transformed:

$$
u \rightarrow u+\frac{3}{2} v, \quad v \rightarrow-v
$$

but the structure of the Hamiltonian itself remains unchanged [1]. Since the RG functions of the problem are completely determined by the structure of the Hamiltonian, the RG equations should be invariant with respect to any transformation conserving this structure [22]. This should be also true for all the expressions relating various universal quantities to each other. If, under $n=2$, we apply the transformation (13) to the magnetization components in Eq. (1), the free-energy expansion remains the same, provided $u_{4}, v_{4}$ are replaced according to Eq. (14) while the sextic coupling constants are transformed as prescribed below:

$u_{6}+\frac{q_{6}}{2}+\frac{v_{6}}{4} \rightarrow u_{6}+q_{6}+v_{6}, \quad \frac{2}{3} q_{6}+v_{6} \rightarrow-\frac{2}{3} q_{6}-v_{6}$.

It means that the following relations between the effective coupling constants should hold:

$$
\begin{aligned}
u_{6}(u, v) & +\frac{q_{6}(u, v)}{2}+\frac{v_{6}(u, v)}{4}=u_{6}\left(u+\frac{3}{2} v,-v\right) \\
& +q_{6}\left(u+\frac{3}{2} v,-v\right)+v_{6}\left(u+\frac{3}{2} v,-v\right) \\
\frac{2}{3} q_{6}(u, v) & +v_{6}(u, v) \\
& =-\frac{2}{3} q_{6}\left(u+\frac{3}{2} v,-v\right)-v_{6}\left(u+\frac{3}{2} v,-v\right)
\end{aligned}
$$

The expansions (10), (11), and (12) do really satisfy these relations. Moreover, consideration of all the remaining limiting cases $[9,11]$ shows that these $R G$ series are in accord with their counterparts obtained earlier for the Ising [23,24] and $O(n)$-symmetric [21] models.

Let us use the expansions just found for the estimation of the universal critical values of the sextic effective coupling constants in the physical case $n=3$. To obtain the numbers of interest from the asymptotic RG series, a proper resummation procedure should be applied. Here we use the Padé-Borel-Leroy resummation technique which demonstrates high numerical effectiveness both for simple (Ising and $O(n)$-symmetric) models $[20,21,25,26, ?]$ and for complicated anisotropic systems preserving their internal symmetries [27]. Since the expansions of quantities depending on two variables $u$ and $v$ are dealt with, the Borel-Leroy transformation is taken in a generalized form:

$$
f(u, v)=\sum_{i j} c_{i j} u^{i} v^{j}=\int_{0}^{\infty} e^{-t} t^{b} F(u t, v t) d t
$$

$$
F(x, y)=\sum_{i j} \frac{c_{i j} x^{i} y^{j}}{(i+j+b) !}
$$

To perform an analytical continuation, the resolvent series

$$
\tilde{F}(x, y, \lambda)=\sum_{n=0}^{\infty} \lambda^{n} \sum_{l=0}^{n} \sum_{m=0}^{n-l} \frac{c_{l, n-l} x^{l} y^{n-l}}{n !}
$$

is constructed which is a series in the powers of $\lambda$ with the coefficients being uniform polynomials in $u, v$ and then Padé approximants $[L / M]$ in $\lambda$ at $\lambda=1$ are used.

With the four-loop RG expansions in hand, we can construct, in principle, three different Padé approximants: [2/1], [1/2], and [0/3]. To obtain proper approximation schemes, however, only diagonal $[\mathrm{L} / \mathrm{L}]$ and neardiagonal Padé approximants should be employed. That is why further we limit ourselves with approximants [2/1] and $[1 / 2]$. Moreover, the diagonal Padé approximant [1/1] will be also dealt with although this corresponds to the usage of the lower-order, three-loop RG approximation.

The algorithm for estimating the universal critical values of sextic effective couplings is as follows. Since, in fact, we have the power series for the ratios $R_{u}=u_{6} / u^{2}$, $R_{q}=q_{6} /(u v), R_{v}=v_{6} / v^{2}$ rather than for $u_{6}, q_{6}, v_{6}$ themselves, we work with the RG series for these ratios. They are resummed in three different ways based on the Borel-Leroy transformation and the Padé approximants just mentioned. The Borel-Leroy integral is evaluated as a function of the shift parameter $b$ under $u=u^{*}, v=v^{*}$. For the cubic fixed point coordinates the values given by the six-loop RG analysis at $n=3$ are adopted [11]: $u^{*}=1.321, v^{*}=0.117$. The optimal value of $b$ providing the fastest convergence of the iteration scheme is then determined. It is deduced from the condition that the Padé approximants employed should give, for $b=b_{\text {opt }}$, the values of $R_{u}\left(R_{q}, R_{v}\right)$ which are as close as possible to each other. Finally, the average over three estimates for $R_{u}\left(R_{q}, R_{v}\right)$ is found and claimed to be a numerical value of this universal ratio.

To demonstrate how such a procedure works, we refer to Table 1 where the results of the corresponding calculations are presented. The empty cells in this Table reflect the fact that for some values of the shift parameter $b$ Padé approximant [1/2] turns out to be spoiled by the "dangerous" poles, i. e. by the poles at positive or small negative $t$. As one can see, for $u_{6}^{*}$ (an integer) $b_{\text {opt }}$, providing a maximal closeness of the estimates given by three working Padé approximants, is equal to 2, while for $q_{6}^{*}$ and $v_{6}^{*} b_{\text {opt }}=3$. So, the results of our four-loop $\mathrm{RG}$ analysis are as follows:

$$
u_{6}^{*}=0.842, \quad q_{6}^{*}=0.175, \quad v_{6}^{*}=0.0108 .
$$

The numerical accuracy of these estimates is fixed both by the accuracy achieved in the course of evaluating the 
cubic fixed point coordinates $u^{*}, v^{*}$ and by the speed of convergence of the iteration procedure employed. The influence of the latter factor may be characterized by the sensitivity of the numerical results with respect to variation of $b$. As is seen from Table 1 , the values of $u_{6}^{*}, q_{6}^{*}$, and $v_{6}^{*}$, averaged over the working Padé approximants, vary by no more than $\pm 0.005, \pm 0.002$, and \pm 0.0001 , respectively, when $b$ runs from 0 to 10 . On the other hand, these error bars are too small to be realistic. Their conservative counterparts may be deduced accepting that the true universal values of renormalized sextic couplings should lie within the intervals of the variation of the estimates given by the leading Padé approximant, namely, by the higher-order pole-free approximant [2/1]. These intervals are easily extracted from Table 1 , they are as follows: $\pm 0.03\left(u_{6}^{*}\right), \pm 0.01\left(q_{6}^{*}\right), \pm 0.001\left(v_{6}^{*}\right)$. Such error bars look quite realistic. Moreover, they are large enough to cover the inaccuracy produced by the uncertainty of the cubic fixed point location. Hence, we adopt these error bars as the final ones.

With the quartic and sextic couplings in hand, we are able to estimate the reduced anisotropy of the sixthorder nonlinear susceptibility. Combining Eqs. (4), (5), and (19), we obtain

$$
\delta^{(6)}=0.102 \pm 0.02
$$

\begin{tabular}{|c|c|c|c|c|c|c|c|}
\hline b & & 0 & 1 & 2 & 3 & 5 & 10 \\
\hline \multirow[t]{3}{*}{$u_{6}^{*}$} & $\overline{\overline{[2 / 1]}}$ & 0.8654 & 0.8514 & $\overline{0.8427}$ & $\overline{0.8368}$ & $\overline{0.8294}$ & $\overline{0.8206}$ \\
\hline & {$[1 / 2]$} & 0.8345 & - & 0.8430 & 0.8384 & 0.8356 & 0.8328 \\
\hline & {$[1 / 1]$} & 0.8097 & 0.8295 & 0.8400 & 0.8466 & 0.8543 & 0.8625 \\
\hline \multirow[t]{3}{*}{$q_{6}^{*}$} & $\overline{[2 / 1]}$ & 0.1834 & 0.1791 & 0.1765 & 0.1747 & 0.1725 & 0.1699 \\
\hline & {$[1 / 2]$} & 0.1729 & 0.1714 & - & 0.1738 & 0.1737 & 0.1729 \\
\hline & {$[1 / 1]$} & 0.1653 & 0.1707 & 0.1736 & 0.1754 & 0.1775 & 0.1797 \\
\hline \multirow[t]{3}{*}{$v_{6}^{*}$} & {$[2 / 1]$} & 0.01168 & 0.01128 & 0.01103 & 0.01086 & 0.01065 & 0.01041 \\
\hline & {$[1 / 2]$} & 0.01062 & 0.01059 & - & 0.01090 & 0.01072 & 0.01064 \\
\hline & {$[1 / 1]$} & 0.00989 & 0.01035 & 01060 & 0.01075 & 0.01093 & 0.01111 \\
\hline
\end{tabular}

Table 1. Numerical estimates for the universal values of sextic effective coupling constants $u_{6}$, $q_{6}$, and $v_{6}$ obtained from the four-loop RG expansions (10), (11), and (12) resummed by the Padé-Borel-Leroy technique using approximants [2/1], [1/2], and [1/1]. The empty cells are due to the "dangerous" poles spoiling corresponding Padé approximants.

Of interest is the role played by the sextic coupling constants in forming the magnitudes of $\chi_{c}^{(6)}, \chi_{d}^{(6)}$, and $\delta^{(6)}$. It may be shown that, in fact, their contrubutions to the sixth-order susceptibility and the anisotropy parameter are very small. Indeed, neglecting $u_{6}, q_{6}$, and $v_{6}$ changes the critical value of $\delta^{(6)}$ by about $4 \%$. In other words, the anisotropy of $\chi^{(6)}$ near $T_{c}$ is fixed practically by the quartic coupling constants only. A similar situation takes place for the higher-order susceptibilities. To demonstrate this, we consider the eighth-order susceptibility $\chi^{(8)}$ for the isotropic (Heisenberg) model. It is expressed via the effective coupling constants:

$$
\chi^{(8)}=-40320 \frac{\chi^{4}}{m^{9}}\left(96 u_{4}^{3}-24 u_{4} u_{6}+u_{8}\right)
$$

Up till now, the universal critical value of the octic coupling constant was determined using the field-theoretical RG approach in three dimensions [21], the biased $\epsilon$ expansion technique [28], and the exact RG machinery. The methods employed lead to the estimates $u_{8}^{*}=0.168$, $u_{8}^{*}=0.36$, and $u_{8}^{*}=0.145$, respectively. All of them are several times smaller than $u_{4}^{*}=0.794$ [19] and $u_{6}^{*}=0.951$ [21]. It means that, because of the big numerical coefficients of the first two terms in brackets of Eq. (21), the contribution of $u_{8}$ to $\chi^{(8)}$ is negligible at criticality. Since the cubic fixed point is located very near the Heisenberg one, the same conclusion is valid for the $3 \mathrm{D}$ cubic model. This enables us to estimate the critical anisotropy of $\chi^{(8)}$ without the calculation of the universal values of the octic couplings. Making in Eq. (21) the substitutions $u_{4} \rightarrow u_{4}+v_{4}, u_{6} \rightarrow u_{6}+q_{6}+v_{6}$ and $u_{4} \rightarrow u_{4}+v_{4} / 3, u_{6} \rightarrow u_{6}+q_{6} / 3+v_{6} / 9$ with $u_{8}$ omitted, we obtain expressions for $\chi_{c}^{(8)}$ and $\chi_{d}^{(8)}$, respectively. Using then the known critical values of the quartic and sextic coupling constants, we obtain the reduced anisotropy of the eighth-order susceptibility:

$$
\delta^{(8)}=\frac{\left|\chi_{c}^{(8)}-\chi_{d}^{(8)}\right|}{\chi_{c}^{(8)}}=0.144 \pm 0.04
$$

The estimates $(6),(20),(22)$ for $\delta^{(4)}, \delta^{(6)}$, and $\delta^{(8)}$ at criticality do not look too small to prevent the detection of the universal cubic anisotropy in physical and computer experiments or by means of a thorough analysis of the high-temperature expansions $[29,30]$. It is well known, however, that the approach of the universal critical regime is controlled by the tiny exponent $\omega \approx 0.01$ [11] making both the experimental study and simulation of the true mode of critical behaviour rather difficult [31]. 
We believe that the results obtained in this paper will help those searching for the cubic asymptotic regimes in real systems and lattice models to choose a proper strategy and the quantities to be measured.

This work was supported by the Russian Foundation for Basic Research (Grant No. 01-02-17048), by the Ministry of Education of the Russian Federation (Grant No. E00-3.2-132), and by the Federal Program "Integratsiya" (Project No. A 0150).
[1] K. G. Wilson, M. E. Fisher, Phys. Rev. Lett. 28, 240 (1972).

[2] D. J. Wallace, J. Phys. C, 6, 1390 (1973).

[3] I. J. Ketley, D. J. Wallace, J. Phys. A 6, 1667 (1973).

[4] A. Aharony, Phys. Rev. B 8, 4270 (1973).

[5] A. I. Sokolov, Fiz. Tverd. Tela 19, 747 (1977) [Sov. Phys. Solid State 19, 433 (1977)].

[6] K. E. Newman, E. K. Riedel, Phys. Rev. B 25, 264 (1982).

[7] I. O. Maier, A. I. Sokolov, Izv. AN SSSR, ser. fiz. 51, 2103 (1987)

[8] N. A. Shpot, Phys. Lett. A 142, 474 (1989).

[9] I. O. Mayer, A. I. Sokolov, B. N. Shalaev, Ferroelectrics 95, 93 (1989).

[10] D. V. Pakhnin, A. I. Sokolov, Phys. Rev. B 61, 15130 (2000).

[11] J. M. Carmona, A. Pelissetto, E. Vicari, Phys. Rev. B 61, 15136 (2000).

[12] H. Kleinert, V. Schulte-Frohlinde, Phys. Lett. B 342, 284 (1995).

[13] H. Kleinert, S. Thoms, Phys. Rev. D 52, 5926 (1995).

[14] H. Kleinert, S. Thoms, V. Schulte-Frohlinde, Phys. Rev. B 56, 14428 (1997).

[15] B. N. Shalaev, S. A. Antonenko, A. I. Sokolov, Phys. Lett. A 230105 (1997).

[16] R. Folk, Yu. Holovatch, T. Yavors'kii, Phys. Rev. B 61, 15114 (2000).
[17] R. Folk, Yu. Holovatch, T. Yavors'kii, Phys. Rev. B 62, 12195 (2000).

[18] A. Pelissetto, E. Vicari, preprint cond-mat/0012164 (2000).

[19] R. Guida, J. Zinn-Justin, J. Phys. A 31, 8103 (1998).

[20] A. I. Sokolov, Fiz. Tverd. Tela 40, 1284 (1998) [Phys. Solid State 40, 1169 (1998)].

[21] A. I. Sokolov, E. V. Orlov, V. A. Ul'kov, S. S. Kashtanov, Phys. Rev. E 60, 1344 (1999).

[22] A. L. Korzhenevskii, Zh. Eksp. Teor. Fiz. 71, 1434 (1976) [Sov. Phys. JETP 44, 751 (1976)].

[23] R. Guida, J. Zinn-Justin, Nucl. Phys. B 489, 626 (1997).

[24] A. I. Sokolov, E. V. Orlov, V. A. Ul'kov, Phys. Lett. A 227, 255 (1997).

[25] G. A. Baker, Jr., B. G. Nickel, D. I. Meiron, Phys. Rev. B 17, 1365 (1978).

[26] S. A. Antonenko, A. I. Sokolov, Phys. Rev. E 51, 1894 (1995).

[27] S. A. Antonenko, A. I. Sokolov, Phys. Rev. B 49, 15901 (1994).

[28] A. Pelissetto, E. Vicari, Nucl. Phys. B 522, 605 (1998).

[29] P. Butera, M. Comi, Phys. Rev. B 56, 8212 (1997); Phys. Rev. B 58, 11552 (1998).

[30] M. Campostrini, A. Pelissetto, P. Rossi, E. Vicari, Phys. Rev. E 60, 3526 (1999); Phys. Rev. B 62, 5843 (2000).

[31] M. Caselle, M. Hasenbusch, J. Phys. A 31, 4603 (1998).

\title{
ЯК РОЗРІЗНИТИ КУБІЧНУ ТА ІЗОТРОПНУ КРИТИЧНУ ПОВЕДІНКУ
}

\author{
Д. В. Пахнін, А. І. Соколов \\ Санкт-Петербурзъкий електротехнічний університет, \\ вул. професора Попова 5, Санкт-Петербург, 197376, Росіл
}

\begin{abstract}
Для тривимірної кубічної моделі проаналізовано нелінійні сприйнятливості четвертого, пюостого й восьмого порядків та розраховано в кубічній нерухомій точпі параметри $\delta^{(i)}$, які характеризують їх зведену анізотропію. Величини анізотропних параметрів становлять: $\delta^{(4)}=0.054 \pm 0.012, \delta^{(6)}=0.102 \pm 0.02$ та $\delta^{(8)}=0.144 \pm 0.04$. Вони вказують, що анізотропну (кубічну) критичну поведінку, яку передбачає детальний ренормалізапійно-груповий аналіз у високих порядках теорії збурень, у принципі можна спостерігати у фізичних та комп'ютерних експериментах.
\end{abstract}

